SELECCIONES MATEMÁTICAS
Universidad Nacional de Trujillo
ISSN: $2411-1783$ (Online)
Vol. $03(01): 8-17$ (2016)

\title{
Semigrupos $n$ - veces Integrados y una aplicación a un problema de tipo Cauchy
}

\section{Semigroups $n$ times Integrated and an application to a problem of Cauchy type}

\author{
Danessa Chirinos Fernández * and Ulices Zavaleta Calderón ${ }^{\dagger}$
}

Received, Mar. 15, 2016

Accepted, Jun. 15, 2016.

DOI: http://dx.doi.org/10.17268/sel.mat.2016.01.02

\section{Resumen}

La teoría de semigrupos n veces integrados es una generalización de los semigrupos fuertemente continuos, la cual fue desarrollada a partir del año 1984, y es muy utilizada para abordar el estudio de la existencia y unicidad de problemas de tipo Cauchy en los que el dominio del operador no es necesariamente denso. En este trabajo se presenta una aplicación de los semigrupos $n$ veces integrados a un problema de viscoelasticidad, el cual es formulado como un problema de Cauchy sobre un espacio de Banach.

Palabras clave. Semigrupo $n$ veces integrado, Problema de Cauchy, viscoelasticidad

\section{Abstract}

The theory of semigroups $n$ times integrated is a generalization of strongly continuous semigroups, which was developed from 1984, and is widely used for the study of the existence and uniqueness of problems such Cauchy in which the operator domain is not necessarily dense. This paper presents an application of semigroups $n$ times integrated into a problem of viscoelasticity, which is formulated as a Cauchy problem on a Banach space presents .

Keywords. Semigroup $n$ times integrated Cauchy problem, viscoelasticity

1. Introducción. Isaac Newton estableció que la evolución de un sistema físico en el tiempo es gobernado por un problema de valor inicial y que los fenómenos físicos son descritas por ecuaciones diferenciales y sus correspondientes problemas de valor inicial.

En general la teoría de semigrupos de operadores se desarrolla en la primera mitad del siglo XX como una formalización de los sistemas autónomo determinístico mediante semigrupo uniparamétrico de transformaciones. Es decir, esta teoría se inicio como un primer lenguaje analítico funcional de las ecuaciones diferenciales parciales, pero pronto también resultas ser una herramienta muy importante en los procesos estocásticos. Alcanzó mayor desarrollo con los trabajos de K. Yosida, E. Hille, R.S. Phillips, I. Miyadera, y, por supuesto W. Feller. Con la publicación del famoso libro: Análisis funcional y semigrupos de E. Hille y R.S. Phillips, la teoría de semigrupos de operadores se ha desarrollado rápidamente tanto del punto de vista teórico como de las aplicaciones, que van desde los clásicos problemas de ecuaciones diferenciales parciales y procesos estocásticos, a problemas menos estándar como ecuaciones integro diferenciales y ecuaciones diferenciales funcionales, la mecánica cuántica, la biología , o en teoría de control.

El desarrollo de esta teoría, específicamente para semigrupo de operadores $n$ veces integrados se debe a los trabajos realizados por Arendt [1], la cual brinda resultados interesantes que aplicaremos en el presente artículo.

En el presente artículo se considera semigrupo de operadores $n$ veces integrados específicamente cuando $n=1$ y su aplicación al modelo de viscoelasticidad

\footnotetext{
* Departamento de Matemáticas de la Universidad Nacional Pedro Ruiz Gallo (dchirinosf@unprg.edu.pe).

${ }^{\dagger}$ Departamento de Matemática de la Universidad Nacional de Trujillo (azavaletaceunitru.edu.pe) This work is licensed under the Creative Commons Attribution-NoComercial-ShareAlike 4.0.
} 


$$
\begin{aligned}
& \rho u_{t t}(t, x)+k u_{t}(t, x)=\psi_{x}(t, x)+h(t, x) \\
& \psi(t, x)=u_{x}(t, x)+\int_{-\infty}^{t} a(t-s) u_{x}(s, x) d s, \quad(t, x) \in \mathbb{R}^{+} \times[0, \tau] \\
& u(t, 0)=u(t, \tau)=0, \quad t \in \mathbb{R}^{+} \\
& u(s, x)=\phi(s, x), \quad s \in \mathbb{R}^{-}
\end{aligned}
$$

donde

$u$ es el desplazamiento

$\psi$ es la fuerza estrés

$h$ es la fuerza externa

$\rho, k \in \mathbb{R}$ son constantes

$\mathbb{R}^{+}=[0, \infty), \mathbb{R}^{-}=(-\infty, 0]$

En este trabajo de investigación se mencionan definiciones y teoremas relacionados sobre semigrupo de operadores $n$ veces integrados, teoremas relacionados a un operador de Hille Yosida, un resultado de perturbación y se prueba el Teorema(4). Se mencionan resultados para garantizar la solución de un problema de Cauchy y problemas integrodiferenciales, con la ayuda de los Teoremas (3)-(6), se obtiene fácilmente otra prueba del Teorema (2.8) debido a Grimmer y Liu [4] (Teorema 7), además se brinda una prueba detallada del Teorema (3.4) debido a Grimmer y Liu [4] (Teorema 8). Se demuestra que el problema de viscoelasticidad tiene única solución.

2. Semigrupo $n$ - veces integrado. Consideremos el espacio de Banach $X$.

Definición 1. Sea $(S(t))_{t \geq 0}$ una familia de operadores lineales acotados sobre $X$ y $n \in \mathbb{N}$. Un semigrupo $(S(t))_{t \geq 0}$ es $n$ veces integrado si

1. $(S(t))_{t \geq 0}$ es fuertemente continua

2. $S(t) S(s)=\frac{1}{(n-1)}\left(\int_{t}^{t+s}(t+s-r)^{n-1} S(r) d r-\int_{0}^{s}(t+s-r)^{n-1} S(r) d r\right)$,

con $s \geq 0, t \geq 0$

Es fácil verificar que la condición (2) de la Definición (1) es equivalente a

$$
S(t) S(s)=\frac{1}{(n-1)} \int_{0}^{s}\left((s-r)^{n-1} S(t+r)-(t+s-r)^{n-1} S(r)\right) d r
$$

La cual es utilizada como definición por otros autores.

Definición 2. Sea $(S(t))_{t \geq 0}$ una familia de operadores lineales acotados sobre $X$.

1. Un semigrupo $n-$ veces integrado $(S(t))_{t \geq 0}$ es no degenerado si $S(t) x=0$ implica $x=0$.

2. Un semigrupo $n$-veces integrado $(S(t))_{t \geq 0}$ es localmente Lipschitz continuo si para todo a $>0$ existe una constante $L=L(a)$ tal que

$$
\|S(t)-S(s)\| \leq L|t-s|
$$

Nota: La condición 2 de la Definición (1) y la condición 1 de la Definición (2) se tiene que $S(0)=0$.

Definición 3. Sean $X$ un espacio Banach, $A: D(A) \subset X \rightarrow X$ un operador lineal y $n \in \mathbb{N}_{0}$. Se dice que A es el generador de un semigrupo $(S(t))_{t \geq 0} n$ veces integrado si existe $M \geq 0, \omega \geq 0$ tal que

1. $\operatorname{abs}(S)<\infty$

2. $(\omega,+\infty) \subset \rho(A)$

3. $R(\lambda, A)=(\lambda I-A)^{-1}=\lambda^{n} \int_{0}^{\infty} e^{-\lambda t} S(t) d t, \quad \operatorname{Re}(\lambda)>\omega$

Nota: Un semigrupo $C_{0}$ es conocido como un semigrupo 0 veces integrado.

Teorema 1. A genera el semigrupo $n$ veces integrado no degenerado $(S(t))_{t \geq 0}$ si se cumple:

$$
x \in D(A) \text { y Ax }=y \text { si y solo si } S(\cdot) x \in C^{1}([0, \infty), X), \quad \frac{d}{d t}(S(t) x)=S(t) y+\frac{t^{n-1}}{(n-1)} x, t \geq 0
$$

Prueba La prueba es directa de la Definición (3). 
El siguiente teorema relaciona un semigrupo $n+1$ veces integrado con el resolvente de su generador.

Teorema 2. Sean $A$ un operador lineal sobre $X, M \geq 0, \omega \in \mathbb{R}$ y $n \in \mathbb{N}_{0}$. Las afirmaciones son equivalentes.

1. $(\omega, \infty) \subset \rho(A) y$

$$
\sup _{k \in \mathbb{N}_{0}} \sup _{\lambda>\omega}\left\|\frac{(\lambda-\omega)^{k+1}}{\lambda^{n}} R(\lambda, A)^{k+1}\right\| \leq M
$$

2. A genera un semigrupo $n+1$-veces integrado $S$ sobre $X$ tal que

$$
\|S(t)-S(s)\| \leq M \int_{s}^{t} e^{\omega r} d r
$$

Para prueba ver Arendt [1].

Nota: Si $A$ es un operador densamente definido, entonces la primera condición del Teorema (2) se satisface si y solo si $A$ genera un semigrupo $n$ veces integrado $S$ y $\|S(t)\| \leq M e^{\omega t}$.

Definición 4 (Condición (H-Y)). Un operador lineal A satisface la condición de Hille- Yosida si existen $M \geq 1$ y $\omega \in \mathbb{R}$ tal que para $\lambda>\omega$ se tiene que

$$
\lambda \in \rho(A), \quad\left\|R(\lambda, A)^{k}\right\| \leq M(\lambda-\omega)^{-k}, k=1,2,3, \ldots
$$

Un operador que satisface la condición de Hille- Yosida es conocido como el operador de Hille-Yosida.

Corolario 1. Las siguientes afirmaciones son equivalentes

a) $\mathcal{A}$ satisface la condición de Hille Yosida

b) $\mathcal{A}$ es el generador de un semigrupo integrado no degenerado tal que

$$
\|S(t)-S(s)\| \leq M \int_{s}^{t} e^{\omega r} d r
$$

Prueba Se sigue directamente del Teorema (5) haciendo $n=0$. $\square$ $[0, \infty)$

Nota: La condición (b) del Corolario (1) indica que $S$ es un operador localmente Lipschitz continuo en

Corolario 2. Sea A es densamente definido sobre $X$. A genera un semigrupo $C_{0}$ sobre $X$ si y solo si A es un operador de Hille Yosida.

Se tiene el siguiente resultado de perturbación debida Arendt [1].

Teorema 3. Si $A$ es un operador de Hille Yosida sobre $X$ y $B \in B(\overline{D(A)}, X)$ entonces $A+B$ es un operador de Hille Yosida.

Prueba Arendt [1].

Teorema 4. Si A es un operador de Hille Yosida sobre $X$ y $B$ genera un semigrupo $C_{0}$ entonces $\operatorname{diag}(A, B)$ es un operador de Hille Yosida.

Prueba En efecto, por hipótesis $B$ genera un semigrupo $C_{0}$ entonces por Corolario (2) el operador $B$ es un operador de Hille- Yosida. Tenemos que $A$ y $B$ satisfacen la condición (H-Y), luego para $\lambda>\omega$

$$
\left[\lambda I-\left(\begin{array}{cc}
A & 0 \\
0 & B
\end{array}\right)\right]\left(\begin{array}{c}
w \\
z
\end{array}\right)=\left(\begin{array}{l}
x \\
y
\end{array}\right) \rightarrow\left(\begin{array}{c}
w \\
z
\end{array}\right)=\left(\begin{array}{cc}
(\lambda-A)^{-1} & 0 \\
0 & (\lambda-B)^{-1}
\end{array}\right)\left(\begin{array}{l}
x \\
y
\end{array}\right)
$$

Considere $C=\operatorname{diag}(A, B)=\left(\begin{array}{cc}A & 0 \\ 0 & B\end{array}\right)$ y los operadores $A$ y $B$ definidos sobre $X$ e $Y$ respectivamente. Considere la norma usual del producto sobre el espacio $X \times Y$. Se tiene que el resolvente de $C$ existe para $\lambda>\omega$, luego para $x \in X, y \in Y$ se tiene 


$$
\begin{aligned}
\left\|R(\lambda, C)^{n}\left(\begin{array}{l}
x \\
y
\end{array}\right)\right\| & =\left\|\left(\begin{array}{c}
(\lambda-A)^{-n} x \\
(\lambda-B)^{-n} y
\end{array}\right)\right\| \\
& =\left\|(\lambda-A)^{-n} x\right\|_{X}+\left\|(\lambda-B)^{-n} y\right\|_{Y} \\
& \leq \frac{M_{1}\|x\|}{(\lambda-\omega)^{n}}+\frac{M_{2}\|y\|}{(\lambda-\omega)^{n}} \\
& =\frac{M\|(x, y)\|}{(\lambda-\omega)^{n}}
\end{aligned}
$$

Por tanto $C$ satisface la condición (H-Y).

3. Problema de Cauchy y ecuaciones integrodiferenciales. Sea el problema de valor inicial

$$
\begin{aligned}
& x^{\prime}(t)=A x(t)+f(t) \\
& x(0)=x_{0}
\end{aligned}
$$

sobre un espacio de Banach $\mathbb{X}, A$ un operador lineal definido sobre $D(A) \subset X$ no necesariamente densamente definido.

Tenemos un Teorema debido a Prato y Sinestrari [2].

Teorema 5. Sea A un operador de Hille Yosida.integrado sobre $X$ para algún $n \in \mathbb{N}$ y $f \in C^{1}(\mathbb{R}, X)$. El problema de Cauchy tiene única solución $x(t)$ si y solo si

$$
x_{0} \in D(A) \quad y \quad A x_{0}+f(0) \in \overline{D(A)}
$$

Sea la ecuación integrodiferencial con retardo

$$
\begin{aligned}
& Z^{\prime}(t)=\mathcal{C} Z(t)+\int_{-\infty}^{t} \Omega(t-s) Z(s) d s+\xi(t), \quad t \geq 0 \\
& Z(s)=\beta(s), \quad s \leq 0
\end{aligned}
$$

sobre un espacio de Banach $\mathcal{P}$, donde $\beta \in W^{1,1}\left(\mathbb{R}^{-}, \mathcal{P}\right)$.

Si hacemos un cambio de variable $m=s-t$ en la integral de (3.2) se obtiene

$$
\int_{-\infty}^{t} \Omega(t-s) Z(s) d s=\int_{-\infty}^{0} \Omega(-m) Z(m+t) d m
$$

Si consideramos la notación $Z_{t}(s)=Z(t+s), s \leq 0$ entonces (3.2) se expresa como

$$
\begin{aligned}
& Z^{\prime}(t)=\mathcal{C} Z(t)+\int_{-\infty}^{0} \Omega(-s) Z_{t}(s) d s+\xi(t), \quad t \geq 0 \\
& Z(s)=\beta(s), \quad s \leq 0
\end{aligned}
$$

Cabe resaltar que

$$
\frac{d Z_{t}(r)}{d r}=\frac{d Z(t+r)}{d r}=\mathcal{C} Z(t+r)+\int_{-\infty}^{0} \Omega(-s) Z_{t+r}(s) d s+\xi(t+r)=\frac{d Z(t+r)}{d t}=\frac{d Z_{t}(r)}{d t}
$$




$$
\begin{aligned}
\operatorname{Si} \widetilde{\Omega}(g)=\int_{-\infty}^{0} \Omega(-s) g(s) d s \text { para } g \in L^{1}\left(\mathbb{R}^{-}, \mathcal{P}\right) \text { entonces } \\
\qquad\left(\begin{array}{c}
Z(t) \\
Z_{t}(s)
\end{array}\right)^{\prime}=\frac{d}{d t}\left(\begin{array}{c}
Z(t) \\
Z_{t}(s)
\end{array}\right)=\left(\begin{array}{c}
\mathcal{C} Z(t)+\widetilde{\Omega}\left(Z_{t}(s)\right)+\xi(t) \\
\frac{d Z_{t}(s)}{d s}
\end{array}\right)
\end{aligned}
$$

Luego, (3.3) sobre $\mathcal{P} \times L^{1}\left(\mathbb{R}^{-}, \mathcal{P}\right)$ se expresa como

$$
\left(\begin{array}{c}
Z(t) \\
Z_{t}(s)
\end{array}\right)^{\prime}=\left(\begin{array}{cc}
\mathcal{C} & \widetilde{\Omega} \\
0 & D_{s}
\end{array}\right)\left(\begin{array}{c}
Z(t) \\
Z_{t}(s)
\end{array}\right)+\left(\begin{array}{c}
\xi(t) \\
0
\end{array}\right), t \geq 0
$$

$$
\left(\begin{array}{l}
Z(0) \\
Z_{0}(s)
\end{array}\right)=\left(\begin{array}{l}
\beta(0) \\
\beta(s)
\end{array}\right)
$$

donde $D_{s}=\frac{\partial}{\partial s}$

Considere el espacio de Banach $X=\mathcal{P} \times L^{1}\left(\mathbb{R}^{-}, \mathcal{P}\right)$ con norma

$$
\|\cdot\|_{X}=\|\cdot\|_{\mathcal{P}}+\|\cdot\|_{L^{1}}, \quad L^{1}=L^{1}\left(\mathbb{R}^{-}, \mathcal{P}\right)
$$

y el operador $\mathcal{A}=\left(\begin{array}{cc}\mathcal{C} & \widetilde{\Omega} \\ 0 & D_{s}\end{array}\right)$ con dominio

$$
D(\mathcal{A})=\left\{\left(\begin{array}{c}
\gamma(0) \\
\gamma
\end{array}\right) \in X: \gamma(0) \in D(\mathcal{C}), \gamma \in W^{1,1}\left(\mathbb{R}^{-}, \mathcal{P}\right)\right\}
$$

Con la ayuda de los Teoremas (3) y (4) obtenemos el siguiente resultado.

Teorema 6. Si $\mathcal{C}$ satisface las condiciones de Hille Yosida sobre el espacio $\mathcal{P}$ y $\Omega \in L^{\infty}\left(\mathbb{R}^{+}, B(\mathcal{P})\right)$ entonces $\mathcal{A}=\left(\begin{array}{cc}\mathcal{C} & \widetilde{\Omega} \\ 0 & D_{s}\end{array}\right)$ satisface las condiciones de Hille Yosida y genera un semigrupo integrado no degenerado localmente Lipschitz continuo sobre X.

Prueba Considere el operador $\mathcal{E}=\left(\begin{array}{cc}\mathcal{C} & 0 \\ 0 & D_{s}\end{array}\right)$ con dominio $D(\mathcal{E})=D(\mathcal{A})$.

Como el operador $D_{s}$ genera un semigrupo $C_{0}$ entonces por corolario 2 se tiene que $D_{s}$ satisface las condición (H-Y). Por hipótesis $\mathcal{C}$ satisface la condición de Hille- Yosida entonces por Teorema (4) se tiene que $\mathcal{E}$ satisface la condición de Hille Yosida.

Por hipótesis $\Omega \in L^{\infty}\left(\mathbb{R}^{+}, B(\mathcal{P})\right)$ entonces el operador $\widetilde{\Omega}$ definido como

$$
\widetilde{\Omega}(h)=\int_{-\infty}^{0} \Omega(-s) h(s) d s, h \in L^{1}\left(\mathbb{R}^{-}, \mathcal{P}\right)
$$

es acotado en $L^{1}\left(\mathbb{R}^{-}, \mathcal{P}\right)$, luego el operador $\mathcal{T}$ definido sobre $\overline{D(\mathcal{E})}=\overline{D(\mathcal{A})}$

$$
\mathcal{T}=\left(\begin{array}{cc}
0 & \widetilde{\Omega} \\
0 & 0
\end{array}\right)
$$

es acotado y por Teorema (3) se tiene que $\mathcal{A}=\mathcal{E}+\mathcal{T}$ es un operador de Hille Yosida. Por Corolario (1), $\mathcal{A}$ genera un semigrupo integrado Lipschitz continuo.

Con la ayuda de los Teoremas (6),(5), se obtiene fácilmente la prueba del Teorema debido a Grimmer y Liu [4]

Teorema 7. Si $\mathcal{C}$ satisface las condiciones de Hille Yosida sobre el espacio $\mathcal{P}, \Omega \in L^{\infty}\left(\mathbb{R}^{+}, B(\mathcal{P})\right)$, $\xi \in C^{1}\left(\mathbb{R}^{+}, \mathcal{P}\right)$ y si

$$
\beta(0) \in D(\mathcal{C}), \quad \beta \in W^{1,1}\left(\mathbb{R}^{-}, \mathcal{P}\right), \quad \mathcal{C} \beta(0)+\xi(0) \in \overline{D(\mathcal{C})}
$$

entonces (3.2) tiene única solución $Z \in C^{1}\left(\mathbb{R}^{+}, \mathcal{P}\right)$.

Prueba En efecto, defina para $t \geq 0$

$$
Y(t)=\left(\begin{array}{c}
Z(t) \\
Z_{t}(s)
\end{array}\right), g(t)=\left(\begin{array}{c}
\xi(t) \\
0
\end{array}\right)
$$


Por hipótesis $\xi \in C^{1}\left(\mathbb{R}^{+}, \mathcal{P}\right), \beta \in W^{1,1}\left(\mathbb{R}^{-}, \mathcal{P}\right)$ y $\beta(0) \in D(\mathcal{C})$ entonces

$$
g=\left(\begin{array}{l}
\xi \\
0
\end{array}\right) \in C^{1}\left(\mathbb{R}^{+}, X\right), D_{s} \beta(s) \in L^{1}\left(\mathbb{R}^{-}, \mathcal{P}\right), Y(0)=\left(\begin{array}{c}
Z(0) \\
Z_{0}(s)
\end{array}\right)=\left(\begin{array}{c}
\beta(0) \\
\beta(s)
\end{array}\right) \in D(\mathcal{A})
$$

Considere $\beta \in W^{1,1}\left(\mathbb{R}^{-}, \mathcal{P}\right)$ tal que $\bar{\Omega}(\beta) \in D(\mathcal{C})$ entonces se tiene que

$$
\mathcal{C} \beta(0)+\xi(0)+\bar{\Omega}(\beta) \in \overline{D(\mathcal{C})}
$$

Luego,

$$
\mathcal{A} Y(0)+g(0)=\left(\begin{array}{c}
\mathcal{C} \beta(0)+\xi(0)+\bar{\Omega}(\beta) \\
D_{s} \beta(s)
\end{array}\right) \in \overline{D(\mathcal{C})} \times L^{1}\left(\mathbb{R}^{-}, \mathcal{P}\right)=\overline{D(\mathcal{A})}
$$

Por Teorema (6), el operador $\mathcal{A}$ genera un semigrupo integrado no degenerado localmente Lipschitz continuo.

Tenemos las hipótesis del Teorema (5): $\mathcal{A}$ genera un semigrupo integrado localmente Lipschitz continuo y $\mathcal{A} Y(0)+g(0) \in \overline{D(\mathcal{A})}$ entonces la ecuación

$$
Y^{\prime}(t)=\mathcal{A} Y(t)+g(t)
$$

tiene una única solución

$$
Y(t)=\left(\begin{array}{c}
Z(t) \\
Z_{t}(s)
\end{array}\right)
$$

Por tanto, $Z(t)$ es la única solución de (3.2).

Los Teoremas $(5,3,4)$ facilitan la prueba de los Teoremas (6) y (7).

Considere la ecuación

$$
\begin{aligned}
x^{\prime}(t) & =A\left(x(t)+\int_{-\infty}^{t} F(t-s) x(s) d s\right)+K x(t)+f(t), \quad t \geq 0 \\
x(s) & =\varphi(s)
\end{aligned}
$$

donde el operador $A$ es no acotado, y los operadores $K$ y $F(t)$ son acotados para $t \geq 0$ sobre un espacio de Banach $\mathcal{X}$, el operador $\varphi$ está referido a las 'historia 'o 'memoria'de la ecuación.

Definición 5. $x:[0, \infty) \rightarrow \mathcal{X}$ es solución de (3.5) si

$$
\begin{aligned}
& \text { 1. } x(t)+\int_{-\infty}^{t} F(t-s) x(s) d s \in D(\mathcal{A}), t \geq 0 \\
& \text { 2. } x \in C^{1}\left(\mathbb{R}^{+}, \mathcal{X}\right) \\
& \text { 3. } x \text { satisface la ecuación (3.5) para } t \geq 0
\end{aligned}
$$

entonces la derivada de $\eta(t)$ es

$$
\frac{d \eta(t)}{d t}=\eta^{\prime}(t)=x^{\prime}(t)+(F * x)^{\prime}(t)=x^{\prime}(t)+\left(F^{\prime} * x\right)(t)+F(0) x(t)
$$

luego la ecuación (3.5) sobre $\mathcal{X} \times \mathcal{X}$ se reformula como

$$
\begin{aligned}
& \left(\begin{array}{l}
x(t) \\
\eta(t)
\end{array}\right)^{\prime}=\left(\begin{array}{cc}
K & A \\
K+F(0) & A
\end{array}\right)\left(\begin{array}{l}
x(t) \\
\eta(t)
\end{array}\right)+\int_{-\infty}^{t}\left(\begin{array}{cc}
0 & 0 \\
F^{\prime}(t-s) & 0
\end{array}\right)\left(\begin{array}{l}
x(s) \\
\eta(s)
\end{array}\right) d s+\left(\begin{array}{l}
f(t) \\
f(t)
\end{array}\right), t \geq 0 \\
& \left(\begin{array}{l}
x(s) \\
\eta(s)
\end{array}\right)=\left(\begin{array}{c}
\varphi(s) \\
\varphi(s)+\int_{-\infty}^{s} F(s-r) x(r) d r
\end{array}\right), s \leq 0
\end{aligned}
$$

Se tiene el siguiente Teorema debido a Grimmer y Liu [4].

Teorema 8. Si las condiciones se cumplen 
1. A satisface $(H-Y)$

2. $K, F(t) \in B(\mathcal{X})$, la derivada fuerte $F^{\prime}(t)$ existe para $t \geq 0, F^{\prime} \in L^{\infty}\left(\mathbb{R}^{+}, B(\mathcal{X})\right), y \varphi \in$ $W^{1,1}\left(\mathbb{R}^{-}, \mathcal{X}\right)$

3. $f:[0, \infty) \rightarrow \mathcal{X}$ continuamente diferenciable

entonces la ecuación (3.5) es bien puesta, es decir, si

$$
\begin{gathered}
w_{0}=\varphi(0)+\int_{-\infty}^{0} F(-s) \varphi(s) d s \in D(A) \\
(K+F(0)) \varphi(0)+A w_{0}+f(0) \in \overline{D(A)}
\end{gathered}
$$

implica que (3.5) tiene única solución $x \in C^{1}\left(\mathbb{R}^{+}, \mathcal{X}\right)$.

Prueba Considere la ecuación (3.6) y defina para algún $t \geq 0$ sobre $\mathcal{P}=\mathcal{X} \times \mathcal{X}$ con norma $\|(\cdot, \cdot)\|=$ $\|\cdot\|+\|\cdot\|$

$$
Z(t)=\left(\begin{array}{c}
x(t) \\
\eta(t)
\end{array}\right), \mathcal{C}=\left(\begin{array}{cc}
K & A \\
K+F(0) & A
\end{array}\right), \Omega(t)=\left(\begin{array}{cc}
0 & 0 \\
F^{\prime}(t) & 0
\end{array}\right), \xi(t)=\left(\begin{array}{c}
f(t) \\
f(t)
\end{array}\right)
$$

entonces (3.6) tiene la forma (3.2).

Por hipótesis $A$ genera un semigrupo integrado $S$ no degenerado localmente Lipschitz continuo entonces por Teorema (1) se tiene que $y \in D(A), A y=w$ si y solo si

$$
S(\cdot) y \in C^{1}([0,+\infty), \mathcal{X}) \text { y } S^{\prime}(t) y=S(t) w+y \rightarrow S(t) w=S^{\prime}(t) y-y=\left(S^{\prime}(t)-I\right) y
$$

Sea $\mathcal{Q}(t)=\left(\begin{array}{cc}t I & S(t)-t I \\ 0 & S(t)\end{array}\right), B=\left(\begin{array}{cc}0 & A \\ 0 & A\end{array}\right), r=\left(\begin{array}{l}x \\ y\end{array}\right) \in \mathcal{X} \times D(A)=D(B)$, entonces $\mathcal{Q}(\cdot) r \in C^{1}([0, \infty), \mathcal{P})$ y

$$
\begin{aligned}
\mathcal{Q}(t) B r & =\left(\begin{array}{cc}
t I & S(t)-t I \\
0 & S(t)
\end{array}\right)\left(\begin{array}{ll}
0 & A \\
0 & A
\end{array}\right)\left(\begin{array}{l}
x \\
y
\end{array}\right) \\
& =\left(\begin{array}{cc}
t I & S(t)-t I \\
0 & S(t)
\end{array}\right)^{\prime}\left(\begin{array}{l}
x \\
y
\end{array}\right)-\left(\begin{array}{l}
x \\
y
\end{array}\right) \\
& =\mathcal{Q}^{\prime}(t) r-r
\end{aligned}
$$

entonces $B$ genera un semigrupo integrado no degenerado $\mathcal{Q}$. Como $S$ es localmente Lipschitz continuo entonces $\mathcal{Q}$ es localmente Lipschitz continuo

$$
\begin{aligned}
\left\|(\mathcal{Q}(t)-\mathcal{Q}(s))\left(\begin{array}{l}
x \\
y
\end{array}\right)\right\|_{\mathcal{K}} & =\|\left(\begin{array}{c}
(t-s) x+\left(\begin{array}{c}
(S(t)-S(s)) y-(t-s) y \\
(S(t)-S(t)) y
\end{array}\right) \|_{\mathcal{K}} \\
\leq L|t-s|\|(x, y)\|_{\mathcal{K}}
\end{array}\right.
\end{aligned}
$$

Por tanto podemos decir que $B=\left(\begin{array}{ll}0 & A \\ 0 & A\end{array}\right)$ genera el semigrupo integrado no degenerado localmente Lipschitz continuo $\mathcal{Q}(t)=\left(\begin{array}{cc}t I & S(t)-t I \\ 0 & S(t)\end{array}\right)$.

Como $B$ es un operador de Hille- Yosida, $K$ y $K+F(0)$ son acotados sobre $\overline{D(B)}$ y por Teorema (3) se tiene que el operador

$$
\mathcal{C}=\left(\begin{array}{cc}
0 & A \\
0 & A
\end{array}\right)+\left(\begin{array}{cc}
K & 0 \\
0 & 0
\end{array}\right)+\left(\begin{array}{cc}
0 & 0 \\
K+F(0) & 0
\end{array}\right)
$$

satisface la condición (H-Y). 
Como $F^{\prime} \in L^{\infty}\left(\mathbb{R}^{+}, B(\mathcal{X})\right)$ y $f:[0, \infty) \rightarrow \mathcal{X}$ es continuamente diferenciable entonces $\Omega \in$ $L^{\infty}\left(\mathbb{R}^{+}, B(\mathcal{P})\right)$ y $\xi \in C^{1}\left(\mathbb{R}^{+}, \mathcal{P}\right)$.

Considere la condición $\beta(s)=Z(s)=\left(\begin{array}{c}x(s) \\ \eta(s)\end{array}\right)=\left(\begin{array}{c}s^{s} \\ \varphi(s)+\int_{-\infty}^{\varphi(s)} F(s-r) \varphi(r) d r\end{array}\right)$ entonces

$$
\begin{aligned}
\mathcal{C} \beta(0)+\xi(0) & =\left(\begin{array}{cc}
K & A \\
K+F(0) & A
\end{array}\right)\left(\begin{array}{c}
\varphi(0) \\
\varphi(0)+\int_{-\infty}^{0} F(-r) \varphi(r) d r
\end{array}\right)+\left(\begin{array}{l}
f(0) \\
f(0)
\end{array}\right) \\
& =\left(\begin{array}{c}
K \varphi(0)+A w_{0}+f(0) \\
(K+F(0)) \varphi(0)+A w_{0}+f(0)
\end{array}\right) \in \mathcal{X} \times \overline{D(\mathcal{A})}=\overline{D(\mathcal{C})}
\end{aligned}
$$

$\mathrm{y}$

$$
\beta(0) \in D(\mathcal{C}), \quad \mathcal{C} \beta(0)+\xi(0) \in \overline{D(\mathcal{C})}
$$

Por otro lado $\beta \in W^{1,1}\left(\mathbb{R}^{-}, \mathcal{P}\right)$ pues $\varphi \in W^{1,1}\left(\mathbb{R}^{-}, \mathcal{X}\right)$

Por tanto por Teorema (7), la ecuación (3.2) tiene única solución $Z=(z, \eta) \in C^{1}\left(\mathbb{R}^{+}, \mathcal{P}\right)$.

Como (3.6) tiene la forma (3.2) entonces $Z(t)=\left(\begin{array}{c}x(t) \\ \eta(t)\end{array}\right)$ es la única solución de (3.6).

Operando el lado derecho de la primera ecuación de (3.6)

$$
\left(\begin{array}{c}
x(t) \\
\eta(t)
\end{array}\right)^{\prime}=\left(\begin{array}{c}
A \eta(t)+K x(t)+f(t) \\
(K+F(0)) x(t)+A \eta(t)+\int_{-\infty}^{t}\left(F^{\prime}(t-s) x(s)\right) d s+f(t)
\end{array}\right), \quad t \geq 0
$$

Como

$$
\begin{aligned}
\eta^{\prime}(t) & =(K+F(0)) x(t)+A \eta(t)+\int_{-\infty}^{t}\left(F^{\prime}(t-s) x(s)\right) d s+f(t) \\
& =K x(t)+A \eta(t)+f(t)+F(0) x(t)+\int_{-\infty}^{t}\left(F^{\prime}(t-s) x(s)\right) d s \\
& =x^{\prime}(t)+\frac{d}{d t} \int_{-\infty}^{t}(F(t-s) x(s)) d s
\end{aligned}
$$

entonces

$$
\eta(t)=x(t)+\int_{-\infty}^{t}(F(t-s) x(s)) d s
$$

Por tanto, $x(t)$ es la única solución de (3.5)

$$
\begin{aligned}
& x^{\prime}(t)=A\left(x(t)+\int_{-\infty}^{t} F(t-s) x(s) d s\right)+K x(t)+f(t), \quad t \geq 0 \\
& x(0)=\varphi(s)
\end{aligned}
$$

4. Aplicación. Utilizaremos los resultados obtenidos arriba para estudiar la ecuación de viscoelasticidad.

$$
\begin{aligned}
& \rho u_{t t}(t, x)+k u_{t}(t, x)=\psi_{x}(t, x)+h(t, x) \\
& \psi(t, x)=u_{x}(t, x)+\int_{-\infty}^{t} a(t-s) u_{x}(s, x) d s, \quad(t, x) \in \mathbb{R}^{+} \times[0, \tau] \\
& u(t, 0)=u(t, \tau)=0, \quad t \in \mathbb{R}^{+} \\
& u(s, x)=\phi(s, x), \quad s \in \mathbb{R}^{-}
\end{aligned}
$$

donde 
$u$ es el desplazamiento

$\psi$ es la fuerza estrés

$h$ es la fuerza externa

$\rho, k \in \mathbb{R}$ son constantes

$\mathbb{R}^{+}=[0, \infty), \mathbb{R}^{-}=(-\infty, 0]$

La primera ecuación es la ecuación del momento lineal y la segunda ecuación relaciona el estrés y la tensión.

Sea $w=u_{t}, \nu=u_{x},{ }^{\prime}=\frac{d}{d t}$ entonces la primera ecuación de (4.1) se escribe como

$$
\begin{aligned}
\left(\begin{array}{c}
\nu^{\prime}(t) \\
w^{\prime}(t)
\end{array}\right)=\left(\begin{array}{c}
\partial_{t}\left(u_{x}(t)\right) \\
u_{t t}(t)
\end{array}\right)= & \left(\begin{array}{c}
\partial_{x}\left(u_{t}(t)\right) \\
\frac{1}{\rho}\left[-k w(t)+\partial_{x}\left(v(t)+\int_{-\infty}^{t} a(t-s) v(s) d s\right)+h(t)\right]
\end{array}\right) \\
= & \left(\begin{array}{cc}
0 & \partial_{x} \\
\frac{\partial_{x}}{\rho} & 0
\end{array}\right)\left[\left(\begin{array}{c}
\nu(t) \\
w(t)
\end{array}\right)+\int_{-\infty}^{t}\left(\begin{array}{cc}
a(t-s) & 0 \\
0 & 0
\end{array}\right)\left(\begin{array}{c}
\nu(s) \\
w(s)
\end{array}\right) d s\right] \\
& +\left(\begin{array}{cc}
0 & 0 \\
0 & -\frac{k}{\rho}
\end{array}\right)\left(\begin{array}{c}
\nu(t) \\
w(t)
\end{array}\right)+\left(\begin{array}{c}
0 \\
\frac{h(t)}{\rho}
\end{array}\right), \quad t \geq 0
\end{aligned}
$$

con $\nu(t)=\nu(t)(x)=\nu(t, x), x \in[0, \tau]$ y de la misma manera para $w$ y $h$. Luego el sistema (4.1) se escribe como

$$
\begin{aligned}
x^{\prime}(t) & =A\left(x(t)+\int_{-\infty}^{t} F(t-s) x(s) d s\right)+K x(t)+f(t), \quad t \geq 0 \\
x(s) & =\varphi(s), \quad s \leq 0
\end{aligned}
$$

donde

$$
A=\left(\begin{array}{cc}
0 & \partial_{x} \\
\frac{\partial_{x}}{\rho} & 0
\end{array}\right), F(t)=\left(\begin{array}{cc}
a(t) & 0 \\
0 & 0
\end{array}\right), K=\left(\begin{array}{cc}
0 & 0 \\
0 & -\frac{k}{\rho}
\end{array}\right), f(t)=\left(\begin{array}{c}
0 \\
\frac{h(t)}{\rho}
\end{array}\right), \varphi(s)=\left(\begin{array}{c}
\phi_{x}(s) \\
\phi_{t}(s)
\end{array}\right)
$$

Observe que las condiciones de borde del modelo de viscoelasticidad $u(t, 0)=u(t, \tau)=0$ implican que $w(0)=w(\tau)=0$. Elegimos el espacio $X=C[0, \tau] \times C[0, \tau]$ de funciones continuas con la norma del supremo para obtener una buena estimación de las soluciones del modelo de viscoelasticidad y de sus derivadas. El dominio del operador principal $A=\left(\begin{array}{cc}0 & \partial_{x} \\ \frac{\partial_{x}}{\rho} & 0\end{array}\right)$ de (4.2)

$$
D(A)=\left\{(\nu, w) \in X: v, w \in C^{1}[0, \tau], w(0)=w(\tau)=0\right\}
$$

no es denso en $X$. La matriz $A$ es similar a la matriz diagonal

$$
\widetilde{A}=\frac{1}{\sqrt{\rho}}\left(\begin{array}{cc}
\partial_{x} & 0 \\
0 & -\partial_{x}
\end{array}\right)
$$

El operador $\widetilde{A}$ es un operador de Hille Yosida sobre $X$ entonces $A$ es un operador de Hille Yosida sobre $X$.

El siguiente teorema garantiza la existencia y unicidad de soluciones del problema de viscoelasticidad.

\section{Teorema 9. Si se cumplen las afirmaciones}

1. $a^{\prime}$ es acotada, uniformemente continua sobre $[0, \infty), a(t) \in B(C[0, \tau])$

2. Para $t \geq 0, h(t, \cdot) \in C[0, \tau]$ y $h_{t}$ es uniformemente continua en $t$ para todo $x \in[0, \tau]$

3. $\phi_{x}, \phi_{t} \in W^{1,1}\left(\mathbb{R}^{-}, C[0, \tau]\right)$

entonces la ecuación (4.1) es bien puesta, es decir, si $\phi$ satisface

$$
\begin{gathered}
\phi_{t}(0, \cdot) \in C^{1}[0, \tau], \quad \phi_{t}(0,0)=\phi_{t}(0, \tau)=0 \\
\phi(0,0)=\phi(0, \tau)=0, \quad \phi_{x}(0, \cdot)+\int_{-\infty}^{0} a(-s) \phi_{x}(s, \cdot) d s \in C^{1}[0, \tau] \\
\left(\phi_{x}(0, \cdot)+\int_{-\infty}^{0} a(-s) \phi_{x}(s, \cdot) d s\right)_{x}+h(0, \cdot)=0, \quad \cdot=0, \cdot=\tau
\end{gathered}
$$


entonces (4.1) tiene única solución u para $(t, x) \in \mathbb{R}^{+} \times[0,1]$.

\section{Prueba}

De la primera hipótesis se tiene que $a^{\prime} \in L^{\infty}\left(\mathbb{R}^{+}, C[0, \tau]\right)$ entonces $F^{\prime} \in L^{\infty}\left(\mathbb{R}^{+}, C[0, \tau]\right)$, como $a(t) \in B(C[0, \tau])$ entonces $F(t) \in B(X)$.

El operador $K$ es constante entonces acotado.

De la segunda hipótesis se tiene que $f$ es continuamente diferenciable.

Y por último de la tercer hipótesis se puede concluir que $\varphi \in W^{1,1}\left(\mathbb{R}^{-}, X\right)$.

Antes del Teorema, se mostró que el operador $A$ satisface las condiciones de Hille- Yosida. Entonces el Teorema (8) garantiza que la ecuación 4.3 tiene una única solución

$$
x=(\nu, w) \in C^{1}\left(\mathbb{R}^{+}, X\right)
$$

Luego, se concluye que (4.1) tiene única solución $u$ para $(t, x) \in \mathbb{R}^{+} \times[0,1]$.

\section{REFERENCES}

[1] Arendt, W., Batty, Ch., Hieber, M. y Neubrander, F. - Vector Valued Laplace transforms and Cauchy problems, Birkhauser, 2010.

[2] Da Prato, G., Sinestrari, E. - Differential operators with non dense domain, Ann. Scuola Norm. Sup. Pisa 14(2), (1987), pp. 285-344.

[3] Desch, W., Grimmer, R., Schappacher, W. - Some considerations for linear integrodifferential equations, J. Math. Anal. Appl. 104, (1984), pp. 219-234.

[4] Grimmer, R. Y LiU, J.- Integrated Semigroups and Integrodifferential Equations, Semigroup Forum Vol. 48, pp. 79-95 (1994)

[5] GRIMMER, R. Y LiU, J.- Integrodifferential Equations with nondensely defined operators, Differential Equations with applications inn Biology, Physics and Engineering, J. Goldstein, F. Kappel and W. Schappacher (eds), Marel Dekker Inc., 1991, 185-199. 